\title{
Determinación de los factores que influyen en la satisfacción del cliente de servicios bancarios de la ciudad de Guayaquil
}

Determination of the factors that influence customer satisfaction of banking services in the city of Guayaquil

\author{
Abarca Erick M. \\ Acosta Andrea M. \\ Bajaña Yanina
}

Fecha de recepción: 02 de mayo de 2020

Fecha de aceptación: 20 de junio de 2020 


\title{
Determinación de los factores que influyen en la satisfacción del cliente de servicios bancarios de la ciudad de Guayaquil
}

\author{
Determination of the factors that influence customer satisfaction of banking
} services in the city of Guayaquil

\author{
Abarca Erick $\mathrm{M}^{1}$, Acosta Andrea $\mathrm{M}^{2}$ y Bajaña Yanina ${ }^{3}$ \\ Como citar: Abarca, E., Acosta, A., Bajaña, Y. (2020). Determinación de los factores que influyen en la satisfacción del cliente de servicios bancarios de la ciudad \\ de Guayaquil. Revista Univsidad de Guayaquil. 131(2), 41-57. DOI: https://doi.org/10.53591/rug.v131i2.1360
}

\section{RESUMEN}

La presente investigación tiene por objetivo examinar la calidad con que se proporcionan los servicios en el sector financiero de la ciudad de Guayaquil, para ello se propone un análisis de los factores que inciden en la satisfacción del cliente de servicios bancarios, de esta manera se pretende reconocer los constructos de mayor relevancia al mismo tiempo que se pretende conocer una herramienta de medición. Para empezar, se realiza una profunda revisión de literatura relacionada con las teorías bases como el SERVQUAL, SERVPERF y el BankServ con el fin de construir una herramienta que ayude a la medición de la satisfacción de la clientela, esto se da lugar mediante una estadística descriptiva y correlacional de la data recolectada. La investigación permite concluir que existen cuatro factores que influyen positivamente en la satisfacción de los clientes de servicios bancarios guayaquileños: calidad del servicio, ambiente, accesibilidad y el factor financiero.

Palabras clave: Satisfacción, clientes, factores, incidencia, servicios bancarios.

\section{ABSTRACT}

The objective of this research is to examine the quality with which services are provided in the financial sector of the city of Guayaquil, for this purpose an analysis of the factors that affect customer satisfaction with banking services is proposed, in this way it is intended Recognize the most relevant constructs while trying to know a measurement tool. To begin with, a thorough

\footnotetext{
${ }^{1}$ Investigador. Universidad Católica Santiago de Guayaquil. Estudiante: erick_m2996@hotmail.com

${ }^{2}$ Investigadora. Universidad Católica Santiago de Guayaquil. Estudiante: ma.andrea_2@hotmail.com

${ }^{3}$ Investigadora. Universidad Católica Santiago de Guayaquil. Docente: yanina.bajana@cu.ucsg.ec
} 
literature review related to basic theories such as SERVQUAL, SERVPERF and BankServ is carried out in order to build a tool that helps to measure customer satisfaction, this is done through descriptive statistics and correlational of the collected data. The research allows us to conclude that there are four factors that positively influence the satisfaction of clients of Guayaquil banking services: quality of service, environment, accessibility and the financial factor.

Keywords: Satisfaction, customers, factors, incidence, banking services.

\section{INTRODUCCIÓN}

La banca puede desaparecer dado el desarrollo del mercado electrónico a nivel mundial por lo que es necesario que la banca vaya innovando y dando seguridad a sus clientes, estableciendo vínculos de confianza, lealtad por lo que la satisfacción del cliente se puede ver afectada por estos factores. Según el Banco Mundial (2018) cerca de 2500 millones de personas en el mundo no tienen acceso a los servicios financieros. Ante esta realidad se pretende incluir a que todas las personas tengan acceso a una cuenta de ahorros, créditos y entre otros productos financieros que puedan satisfacer necesidades de las personas.

Considerando que la competencia en el sector bancario se hace cada día más importante es imperante buscar la diferenciación en la mejora de servicios que alcancen una mayor satisfacción de los clientes. Teniendo en cuenta que la satisfacción del cliente es considerada como un elemento fundamental para alcanzar la competitividad del sector bancario. East (1997) argumentó que, si un cliente queda satisfecho con un servicio recibido, la probabilidad de que vuelva a utilizar tal servicio incrementa. Si un cliente satisfecho tiene el potencial de influir en 100 nuevos clientes, un cliente insatisfecho puede influir en 1000 posibles clientes (Sangwan, 2009).

En la actualidad, la banca privada de Ecuador ha visto más complicado el proceso de fidelización y de satisfacción al cliente (Asobanca, 2016). Esto debido a que las necesidades de los clientes se vuelven más exigentes a través del tiempo. La globalización ha dado lugar a las actuales aperturas de mercado, permitiendo al mismo tiempo el ingreso de capital extranjero a través de la banca privada, lo que a su vez ha impulsado a la banca nacional a mejorar sus servicios mediante la inversión en temas de innovación tecnológica de seguridad y mejora de sus servicios para poder satisfacer adecuadamente a sus usuarios. 
La presente investigación tiene como finalidad identificar los principales factores que impactan y su nivel de influencia sobre la satisfacción del cliente de servicios bancarios de la ciudad de Guayaquil.

\section{Justificación}

Mediante la presente investigación se pretende generar conocimiento con herramientas que permitan mejorar la satisfacción del cliente de servicios bancarios

La presente investigación tiene como finalidad aportar académicamente al desarrollo de herramientas que permitan el estudio del complejo constructo denominado "satisfacción del cliente". Actualmente, en Ecuador, la literatura relevante sobre este tema es escasa; son pocos los autores que han dedicado horas de investigación al análisis de la satisfacción del cliente, no solo para el sector financiero sino para todo tipo de servicios mencionables. Por tal motivo, esta investigación pretende ampliar la literatura existente acerca de este tema, esperando aportar de cierto modo en otras investigaciones relacionadas.

\section{Objetivo General}

Evaluar los factores que influyen en la satisfacción del cliente de servicios bancarios de la ciudad de Guayaquil.

\section{Objetivos Específicos}

1. Establecer la fundamentación teórica y modelos de medición de satisfacción de clientes de servicios bancarios.

2. Seleccionar la metodología apropiada para levantar la información pertinente para la evaluación de los factores relacionados a la satisfacción de clientes, en el sector bancario.

3. Evaluar los factores que impactan sobre la satisfacción del cliente de servicios bancarios de la ciudad de Guayaquil

\section{Hipótesis}

Dado los objetivos anteriormente expuestos, se plantea las siguientes hipótesis:

- H1. El factor de la calidad del servicio incide sobre los niveles de satisfacción del cliente de servicios bancarios de la ciudad de Guayaquil. 
- H2. El factor de higiene/ambiente incide sobre los niveles de satisfacción del cliente de servicios bancarios de la ciudad de Guayaquil.

- H3. El factor de involucramiento incide sobre los niveles de satisfacción del cliente de servicios bancarios de la ciudad de Guayaquil.

- H4. El factor accesibilidad de los servicios incide sobre los niveles de satisfacción del cliente de servicios bancarios de la ciudad de Guayaquil.

- H5. El factor financiero incide sobre los niveles de satisfacción del cliente de servicios bancarios de la ciudad de Guayaquil.

\section{REVISIÓN DE LITERATURA}

Una de las estrategias fundamentales para generar rentabilidad y sostenibilidad de las empresas en el tiempo ha sido la satisfacción del cliente por lo que este constructo ha sido ampliamente abordado en el marketing y ventas. La satisfacción del cliente es una respuesta cognitiva, un juicio evaluativo, respuesta afectiva, una comparación de percepciones y expectativas (Churchill \& Surprenant, 1982; Day, 1984; Oliver, 1997; Swan, Trawick \& Carroll, 1980). Diversos autores como Bachelet, 1992; Halstead, Hartman \& Schmidt, 1994 definen la satisfacción del cliente como esa manifestación emocional que tiene el consumidor al recibir un servicio y compara entre lo que esperaba y lo que recibió.

Se realiza un análisis a profundidad de las diversas teorías que engloban la medición de la satisfacción del consumidor y que permiten ampliar el espectro de conocimiento referente a este constructo con la finalidad de crear el instrumento de medición.

\section{Teorías de medición de la satisfacción del cliente}

Se abordan distintos modelos de medición de la calidad se inicia con el modelo denominado Modelo de la Calidad Percibida del servicio en el que se compara las expectativas que tiene el consumidor sobre un servicio contra la percepción real del servicio recibido (Grönroos,1984). Este autor propone que las expectativas del consumidor vienen dadas por factores como las tradiciones y las ideologías que tiene sobre el servicio a recibir, pero además también se conforman por las expectativas fruto de las experiencias previas con servicios similares y las "promesas" realizadas por los ofertantes. Mientras que la calidad percibida proviene de la imagen dada del servicio que comprende dos dimensiones como lo menciona este autor que son: (a) la calidad técnica del 
servicio que es aquello que recibe un cliente al utilizar un determinado servicio y (b) la calidad funcional del servicio que se refiere en la forma en que un cliente recibe un servicio. Este modelo es considerado como uno de los precursores de otros que dan paso a la medición de la satisfacción del cliente como el SERVQUAL y el SERVPERF, el mismo que propicia evaluar la dimensión de mayor impacto sobre la satisfacción del consumidor, de acuerdo a investigadores como Zeithaml, Parasuraman \& Berry (1988) y a Cronin y Taylor (1992).

Por otra parte, se plantea el modelo SERVQUAL propuesto por Zeithaml et al. (1988) el cual faculta comparar las expectativas de un servicio evaluadas a través de comentarios de terceros, necesidades y experiencias pasadas en relación con las percepciones que tiene el consumidor con tal servicio, bajo cinco dimensiones que son: confiabilidad, seguridad, sensibilidad, empatía y tangibilidad. Debido a diversas críticas realizadas al modelo SERVQUAL de Zeithaml et al. (1988). Es pertinente mencionar también a autores como Cronin y Taylor (1992) quienes desarrollaron un nuevo modelo para medir la satisfacción del cliente, sentando sus basado en determinados parámetros del Service Quality, proponiendo como principal diferencia entre el modelo SERVQUAL del SERVPERF es que este último se enfoca en la valoración del desempeño del servicio recibido, eliminando las expectativas que tienen los clientes previos a la recepción del servicio. Al igual que el modelo SERVQUAL, el SERVPERF de Cronin \& Taylor (1992) considera las mismas cinco dimensiones.

Fundamentándose en los modelos SERVQUAL y SERVPERF, Avikran (1994) desarrolló el modelo Bank Service o BANKSERV, planteado para evaluar los niveles de satisfacción de los clientes específicamente para servicios bancarios en Australia. En el modelo BANKSERV se propuso la evaluación de seis dimensiones, donde tres de ellas son del modelo SERVQUAL que son: empatía, confiabilidad y sensibilidad; las otras dimensiones que añadió el autor son: comunicación, acceso a los ATMs y conducta de los empleados. Posterior a la formulación de la prueba piloto, el cuestionario se concretó en cuatro dimensiones que son: (a) Conducta de los empleados del banco (b) Credibilidad; (c) Comunicación; (d) Acceso a los ATMs (Cajeros).

Con la información recopilada de los modelos de evaluación de la satisfacción del cliente, Bahia y Nantel (2000) desarrollaron el modelo Bank Service Quality o BSQ en función de las siete Ps del marketing: producto, plaza, proceso, participantes, precio, promoción y posición (lugar físico). Al igual que el SERVQUAL de Zeithaml et al. (1988) el Bank Service Quality tiene la finalidad de comparar las expectativas del consumidor sobre el servicio ideal que debería ofrecer el banco 
contra los servicios realmente ofrecidos por un determinado banco. Luego de diversos tipos de análisis estadístico de consistencia interna, se redujeron las dimensiones evaluadas de 15 a tan solo seis que son: seguridad, accesibilidad a los servicios, precios, tangibilidad, portafolio de servicios y confiabilidad del banco., siendo distintivo el hecho de que dentro de la dimensión \#1 de las seis resultantes se incluyeran todas las variables evaluadas en el cuestionario SERVQUAL.

El modelo SERVQUAL ha sido utilizada por diferentes autores los cuales concluyen que es un buen modelo para analizar la satisfacción del cliente (Awan et al., 2011; Hennayake, 2017; Jabnoun \& Khalifa, 2005; Kaur et al., 2012; Paul \& Srivastav, 2016; Subhashini \& Preetha, 2018). Por otra parte, Gupta y Dev (2012), determinaron que los factores con mayor significancia con respecto a la satisfacción del cliente en bancos de la India son: calidad del servicio, ambiente e higiene y participación del cliente; pero mencionan que los factores analizados pueden cambiar según el contexto.

En un estudio realizado por Ushanta, Wijeratne y Samantha (2014) en Sri Lanka concluyeron que existe una relación positiva entre la calidad del servicio y la satisfacción del cliente, además consideraron que la escala SERVPERF es adecuada para su medición. Por otro lado, otras investigaciones corroboran que el factor de ambiente es uno de los que tienen mayor incidencia en la satisfacción del cliente (Famiyeh, Asante-Darko \& Kwarteng, 2018; Jabnoun \& Khalifa, 2005; Saleh et al., 2017; Torres \& Luna, 2017; Yilmaz, Ari \& Gürbüz, 2018).

En función de la revisión literaria de las teorías anteriormente explicadas, Gupta y Dev (2012) elaboran un cuestionario para la medición de la satisfacción del cliente con las variables de calidad del servicio, accesibilidad, involucramiento, ambiente y el factor financiero. Para el estudio se utiliza el cuestionario de Gupta y Dev (2012) como base y se adjuntó preguntas al constructo de satisfacción del cliente del cuestionario validado por Saleh, Keating y Gaur (2017).

\section{Conceptualizaciones}

Una vez que se analizó las teorías que determinan cuales son las variables que deben ser analizadas para estudiar el constructo "satisfacción del cliente", cabe destacar que de todas las que fueron presentadas, en el presente estudio se analizará la calidad del servicio, el factor ambiental, el involucramiento del cliente, la accesibilidad a los servicios bancarios y el factor financiero como principales componentes que impactan sobre la satisfacción del usuario de servicios bancarios. 
Para el presente estudio, se utilizará la definición de calidad del servicio brindada por Zeithaml et al. (1988), en la que el concepto de calidad se refiere a la calidad percibida, es decir, al juicio del consumidor sobre la excelencia y superioridad de un producto. En esta definición, básicamente se compara la calidad percibida de un determinado producto o servicio con las expectativas del consumidor, considerando las cinco variables del modelo SERVQUAL antes explicadas (confiabilidad, seguridad, sensibilidad, empatía y tangibilidad).

Con respecto al factor de higiene, o también conocido como factor ambiental, de acuerdo a Gupta y Dev (2012) este factor referencia a aquella parte tangible de los servicios ofrecidos. Para estos investigadores, a pesar de la naturaleza intangible de los servicios bancarios ofrecidos, la satisfacción de los usuarios de tales servicios tiende a ser influenciada por las instalaciones físicas del banco como las máquinas utilizadas para las transacciones, equipamiento en cada sucursal bancaria, y el desempeño de la misma para el cumplimiento de funciones. Para fines aclaratorios, se define al factor higiene/ambiental como todos aquellos aspectos físicos que de alguna manera ejercen influencia sobre la entrega del servicio al cliente, afectando positiva o negativamente su satisfacción.

Con respecto al factor involucramiento del cliente, se asevera que este proviene del factor denominado “Comunicación” del Modelo Bank Service, BANKSERV de Avikran (1994). En este modelo, tal factor se define como la capacidad de la empresa de mantener comunicados a los clientes de toda la información necesaria acerca de sus servicios, así como la capacidad de satisfacción de necesidades de la clientela a través de una comunicación exitosa y asesoramiento financiero adecuado. Por otra parte, a pesar de no ser mencionados en las investigaciones base acerca de la satisfacción del cliente, el factor accesibilidad aparece en la mayoría de estudios enfocados al estudio de la satisfacción de los usuarios de servicios bancarios; ejemplos de estos estudios son el BANKSERV, el Bank Service Quality y el China el Bank Service Quality, antes mencionados. En investigaciones como la de Penchansky y Thomas (1981) el factor de accesibilidad se define como la relación existente entre la localización de entrega del servicio y la ubicación actual de los clientes, tomando en cuenta la transportación del cliente, los costos de transportación para obtener el servicio, el tiempo y las distancias que este debe recorrer para satisfacer sus necesidades. Para esta investigación, se define el factor accesibilidad (a los servicios bancarios) como definida como la facilidad de uso del servicio que tienen los clientes a los 
servicios bancarios correspondiente a través de cajeros y la flexibilidad horaria de las sucursales bancarias.

Con respecto a la última variable independiente del análisis; el factor financiero se define como aquella parte que el cliente o usuario final está dispuesto a cancelar por el uso de un determinado servicio, en este caso por el uso de todos los servicios financieros promocionados por el banco. Finalmente, como ya fue explicado anteriormente, la única variable dependiente de los otros factores anteriormente definidos es la satisfacción del cliente de los servicios bancarios. Este constructo puede llegar a tener diversas definiciones dependiendo del enfoque que se le dé y dependiendo del contexto en el cual se lo analice.

En la actualidad, de acuerdo con Tse y Wilton (1988) "la satisfacción del cliente es la respuesta del consumidor a la evaluación de la discrepancia percibida entre las expectativas anteriores (o alguna norma de rendimiento) y el rendimiento real del producto/servicio tal como se percibe después de su consumo”. (p.204)

\section{METODOLOGÍA}

La presente investigación plantea en un enfoque cuantitativo utilizando como herramienta la encuesta para la recolección de datos en un semestre del año 2019, su corte de tiempo es de tipo transversal, al haber sido aplicadas en un punto específico del tiempo, además no hubo manipulación de variables por lo que este estudio es no experimental (Hernández, Fernández y Baptista, 2010). El método de esta investigación es hipotético-deductivo ya que se establecen una o varias hipótesis a partir de la revisión literaria, para posteriormente corroborar la certeza de tales hipótesis a través de análisis estadísticos (Rodríguez \& Pérez, 2017). De acuerdo a Cortés e Iglesias (2005) el alcance de esta investigación es descriptivo - correlacional, ya que se parte de una descripción de características concernientes con los factores a estudiarse y analizar las relaciones que pudiesen existir entre los constructos propuestos.

\section{Instrumento de medición}

Un cuestionario desarrollado por Gupta y Dev (2012) se utilizó como instrumento de medición al cual se agregaron preguntas del constructo de satisfacción al cliente elaborado por (Saleh, Quazi, Keating \& Gaur, 2017). El instrumento consta de dos secciones, la primera sección reúne información demográfica y la segunda sección está formada por 34 ítems y se encuentran 
agrupados en seis constructos que son: calidad del servicio, ambiente/ higiene, involucramiento, accesibilidad y factores financieros como variables independientes y satisfacción al cliente como la única variable dependiente; se hizo uso de la escala LIKERT del uno al cinco. La población considerada es finita formada por la todos los individuos de la ciudad de Guayaquil que poseen una cuenta de ahorros o corriente, a partir de eso se definió la muestra que estuvo compuesta únicamente por clientes que tengan al menos uno de los servicios anteriormente mencionados en alguna institución financiera de la ciudad de Guayaquil, alcanzando la recolección de 271 encuestas, considerando un nivel de confianza del $94 \%$ y un margen de error del $6 \%$.

El propósito del análisis de la información es la evaluación de la existencia o ausencia de relación entre la satisfacción del cliente y los factores de calidad del servicio, ambiente, involucramiento, accesibilidad y financieros. Para el correspondiente análisis e interpretación de datos se utilizó el programa Microsoft Excel y el software SPSS 23 que dieron lugar a la prueba ANOVA.

Como proceso de validación del cuestionario se procedió primero con la doble traducción del cuestionario, ya que el original está en idioma inglés, luego seguido se realizó un grupo focal con expertos que permitieron adaptar las preguntas para que se puedan comprender de forma clara en el contexto de estudio; posteriormente se realizó un levantamiento de da a 30 personas para realizar una prueba piloto y así calcular el alfa de Cronbach, y poder validar el cuestionario; el cual se midió con una escala LIKERT del uno al cinco, donde uno es totalmente desacuerdo, dos en desacuerdo, tres ni de acuerdo ni en desacuerdo, cuatro de acuerdo y cinco totalmente de acuerdo.

\section{RESULTADOS}

Se realizó al análisis del coeficiente Alfa de Cronbach $(\alpha)$ de toda la prueba, el cual mostró un valor de 0.959 considerando los 34 ítems analizados corroborando una sólida consistencia interna de la prueba, superando incluso el Alfa obtenido en el estudio base de Gupta y Dev (2012) el cual presentó un Alfa de 0.907, por lo que se confirma su validez ya que según Hernández et al. (1989) el mínimo aceptable es 0.70 .

\section{Variables Demográficas}

En la Tabla 1 se pueden evidenciar resultados de las variables demográficas, donde el 32\%, de los clientes encuestados pertenecen al rango de edad de 18 y 25 años, seguido con un 28,00\% los 
clientes que se encuentran en una edad entre 51 y 69 años, por otra parte, un $16 \%$ de los encuestados se encuentran entre los 26-32 y los 33-41 y finalmente solo un 8\% entre los 51-69. Por otra parte, en relación con el género se observa que el 59\% de clientes encuestados fueron del género femenino y $41 \%$ fueron del género masculino. En relación con el nivel académico, el 72\%, poseen estudios universitarios, $14 \%$ poseen posgrados, $13 \%$ solo estudios secundarios y un $0.37 \%$ tan solo estudios primarios. Adicionalmente, se puede apreciar que el 77\% tienen una cuenta de Ahorros, $14 \%$ una cuenta corriente y solo $9 \%$ poseen ambos tipos de cuenta.

\section{Tabla 1}

Variables Demográficas

\begin{tabular}{lcc}
\hline Edad & Cant. & \% \\
\hline $18-25$ & 86 & $32 \%$ \\
$26-32$ & 44 & $16 \%$ \\
$33-41$ & 44 & $16 \%$ \\
$42-50$ & 22 & $8 \%$ \\
$51-69$ & 75 & $28 \%$
\end{tabular}

Género

Femenino

$159 \quad 59 \%$

Masculino

$112 \quad 41 \%$

Nivel de estudios

Estudios primarios

$1 \quad 0,37 \%$

Estudios secundarios

$35 \quad 13 \%$

Estudios universitarios (pre-grado)

$195 \quad 72 \%$

Estudios Pos- universitarios (master, doctorado)

$40 \quad 15 \%$

Tipo de cuenta

Ahorros

$210 \quad 77 \%$

Corriente

$37 \quad 14 \%$

Ambas

$24 \quad 9 \%$

\section{Regresión}

Mediante un análisis de regresión se pudo determinar el coeficiente de correlación de Pearson el mismo que mide la relación que existe entre la variable dependiente y las variables independientes. Como se puede apreciar en la Tabla 2, el coeficiente de correlación es de $\mathrm{r}=0.85$ lo que permite inferir una correlación positiva considerable (Hernández, Fernández \& Sampieri, 2010). Ante estos hallazgos se determina que los factores de calidad, ambiente, involucramiento, accesibilidad y financieros guardan una relación fuerte con la satisfacción del cliente. Seguido se hizo el análisis 
del coeficiente de determinación el mismo que hace referencia a cuanto la ecuación o variables independientes explican a la depndiente. Para el estudio el coeficiente de determinación o $\mathrm{r}^{2}=.73$; un valor que para Cresswell (2005) ofrece una buena predicción de una variable con respecto de otra, ante esto se concluye que los factores como calidad, ambiente, involucramiento, accesibilidad y financieros explican en un 73,42\% de la satisfacción del cliente.

Tabla 2

Resultados de la Prueba ANOVA

\begin{tabular}{ll}
\hline \multicolumn{2}{c}{ Estadísticas de Regresión } \\
\hline Coeficiente $\mathrm{r}$ & 0.8568876 \\
R cuadrado & 0.7342563 \\
R cuadrado ajustado & 0.7292233 \\
Error Estándar & 0.4349759 \\
Observaciones & 271 \\
\hline
\end{tabular}

ANOVA

\begin{tabular}{|c|c|c|c|c|c|}
\hline & $d f$ & $S S$ & $M S$ & $F$ & $\begin{array}{c}\text { Significancia } \\
F\end{array}$ \\
\hline Regresión & 5 & 138.0126852 & 27.60254 & 146.4402 & $7.81666 \mathrm{E}-74$ \\
\hline Residual & 265 & 49.9498662 & 0.188490 & & \\
\hline Total & 270 & 187.9625514 & & & \\
\hline
\end{tabular}

\begin{tabular}{lcccccc}
\hline & Coeficientes & $\begin{array}{c}\text { Error } \\
\text { Estándar }\end{array}$ & $\begin{array}{c}\text { Estadístico } \\
t\end{array}$ & Valor P & $\begin{array}{c}\text { Limite } \\
\text { Inferior 95\% }\end{array}$ & $\begin{array}{c}\text { Limite Superior } \\
95 \%\end{array}$ \\
\hline Intercepto & -0.1865746 & 0.174430345 & -1.06962 & 0.285766 & -0.53002629 & 0.1568771 \\
Calidad & 0.24206869 & 0.060794783 & 3.981735 & $8.85 \mathrm{E}-05$ & 0.122364337 & 0.361773 \\
Ambiente & 0.42974703 & 0.052791633 & 8.140438 & $1.57 \mathrm{E}-14$ & 0.325800809 & 0.5336933 \\
Involucram. & -0.0310817 & 0.0378189 & -0.82186 & 0.411901 & -0.1055468 & 0.043383 \\
Accesibilidad & 0.21665124 & 0.044485158 & 4.870192 & $1.92 \mathrm{E}-06$ & 0.129060392 & 0.3042421 \\
Financiero & 0.22142771 & 0.035119214 & 6.30503 & $1.2 \mathrm{E}-09$ & 0.152278316 & 0.2905771 \\
\hline
\end{tabular}

\section{Análisis de Hipótesis}

Los resultados de la prueba ANOVA revelan que los cinco factores analizados si inciden en la satisfacción del cliente, ya que según la prueba Fisher F=7,8166E-74 es menor al nivel de 
significancia de investigación que es de 0.06; por lo tanto, se rechaza la hipótesis nula que refiere a que los factores analizados no inciden en la satisfacción del cliente.

H1. El factor de la calidad del servicio incide sobre los niveles de satisfacción del cliente de servicios bancarios de la ciudad de Guayaquil.

Con respecto a la primera hipótesis planteada para esta investigación que abarca el factor calidad, el cual tiene un $\mathrm{p}<0.006$ menor al nivel de significancia de 0.06 , por lo que se acepta la hipótesis alternativa, es decir la calidad del servicio si tiene incidencia sobre la satisfacción del cliente de los servicios bancarios de la ciudad de Guayaquil. Por cada unidad del factor calidad del servicio que se aumente, manteniendo las otras variables constantes, la satisfacción del cliente aumentará proporcionalmente un valor de 0.24 .

H2. El factor de higiene/ambiente incide sobre los niveles de satisfacción del cliente de servicios bancarios de la ciudad de Guayaquil.

La siguiente hipótesis referente al factor ambiente e higiene, revela un $\mathrm{p}<0.006$ en la prueba ANOVA, por lo que al ser menor que el nivel de significancia de 0.06 se puede concluir que se acepta la hipótesis alternativa, por lo tanto, el factor ambiente e higiene incide sobre la satisfacción del cliente de los servicios bancarios de la ciudad de Guayaquil. Por cada unidad del factor ambiente que se aumente, manteniendo las otras variables constantes, la satisfacción del cliente aumentará proporcionalmente un valor de 0.42 .

H3. El factor de involucramiento incide sobre los niveles de satisfacción del cliente de servicios bancarios de la ciudad de Guayaquil.

El siguiente factor analizado es sobre el involucramiento de los clientes con el banco, según su coeficiente de - 0,02 indica que ha medida que más se involucra al cliente menos satisfechos están los clientes. Como se puede observar en la Tabla 2, el P- value es de 0.41; es decir mayor al nivel de significancia de 0.06; ante esto se concluye que el factor involucramiento no incide en la satisfacción del cliente de los servicios bancarios de la ciudad de Guayaquil.

H4. El factor accesibilidad de los servicios incide sobre los niveles de satisfacción del cliente de servicios bancarios de la ciudad de Guayaquil. 
El siguiente factor analizado es sobre la accesibilidad de los clientes con respecto a las los cajeros automáticos, sucursales del banco, los horarios de atención; el cual muestra un $\mathrm{p}<0.006$ en la prueba ANOVA, siendo este menor al nivel de significancia de 0.06. Por lo tanto, el factor accesibilidad si incide con respecto a la satisfacción del cliente de los servicios bancarios de la ciudad de Guayaquil. La satisfacción del cliente aumentará proporcionalmente un valor de 0.21 por cada unidad del factor accesibilidad que se aumente, manteniendo las otras variables constantes,

H5. El factor financiero incide sobre los niveles de satisfacción del cliente de servicios bancarios de la ciudad de Guayaquil.

Finalmente, el último factor que se refiere al financiero trata sobre los costos, tasas de interés, cargos por servicios; como señala en la Tabla 2 tiene un $\mathrm{p}<0.006$ el cual es menor al nivel de significancia de 0.06. Por lo tanto, se concluye que el factor financiero incide en la satisfacción del cliente de los servicios bancarios de la ciudad de Guayaquil. La satisfacción del cliente aumentará proporcionalmente un valor de 0.22 , por cada unidad del factor financiero que se aumente, manteniendo las otras variables constantes. A partir de esta información se genera la ecuación que sirve para explicar el $73 \%$ de la satisfacción del cliente de servicios financieros en Guayaquil se expresa de la siguiente manera:

$$
\text { Satisfacción del cliente }=-0.18+0.24 \mathrm{C}+0.42 \mathrm{H}+0.21 \mathrm{~A}+0.22 \mathrm{~F}
$$

Siendo:

$\mathrm{C}=$ Calidad del Servicio

$\mathrm{H}=$ Higiene/Ambiente

A= Accesibilidad

$\mathrm{F}=$ Factor Financiero

Adicionalmente, se puede interpretar el intercepto negativo de la ecuación como el hecho de que los clientes que hacen uso de servicios bancarios en la ciudad de Guayaquil están intrínsecamente insatisfechos con el servicio ofrecido por los bancos de la ciudad. 


\section{CONCLUSIONES}

Acorde a la investigación y las relaciones establecidas se puede evidenciar en base a los hallazgos de los distintos testeos estadísticos que la satisfacción del cliente depende de la calidad del servicio, ambiente e higiene, accesibilidad y factores financieros. Se concluye que la población objeto de estudio revelan que las variables independientes explican en un 73\% el nivel de satisfacción de los servicios financieros. No obstante, el factor de involucramiento no tiene incidencia sobre la satisfacción de los clientes. Adicionalmente, realizando el análisis en multigrupos por el criterio del nivel de estudios y el rango de edades, el factor de calidad de servicios puede diferir, ya que en las personas que su nivel de estudios es hasta nivel secundario este factor no tiene incidencia en su satisfacción, al igual que en personas que se encuentran en un rango de edad de 18 a 32 años que casi no tiene incidencia. Distinto escenario ocurre con el factor de ambiente que se refiere a los elementos tangibles del banco como los equipos que tenga el banco sean modernos, las instalaciones físicas sean las adecuadas, la vestimenta de los empleados es la correcta, que el ambiente en general es bastante bueno; es considerado el factor que tiene una mayor significancia sobre la satisfacción de los clientes. Se puede decir que el instrumento permitió identificar los factores que influyen en la satisfacción del cliente que reciben servicios del sector bancario, pero que este de igual manera como lo señalan otros autores puede variar según el contexto.

\section{RECOMENDACIONES}

Se sugiere para futuras investigación ampliar el tamaño que la utilizada para el presente estudio, o considerar realizarla en otros tipos de contextos. A pesar de que la investigación realizada se elaboró considerando un $94 \%$ de confiabilidad y $6 \%$ de error, se sugiere el incremento del número de la muestra para la reducción del margen de error, con la finalidad de obtener resultados más certeros. Además, se sugiere ampliar la revisión literaria y consultar a expertos en temas de satisfacción del cliente de servicios bancarios con la finalidad de establecer uno o varios constructos adicionales que permitan explicar el 26,58\% faltante de la satisfacción de clientes de servicios bancarios (financieros) en la ciudad de Guayaquil expresada a través de la ecuación de satisfacción al cliente que ha sido propuesta. Finalmente, se recomienda el descarte del factor denominado "involucramiento del cliente", puesto que quedó demostrado en la presente investigación que tiene no tiene incidencia sobre la satisfacción del cliente, por lo tanto, los ítems de este factor dentro del cuestionario deberían ser descartados al realizar futuras investigaciones. 


\section{REFERENCIAS}

Avikran, K. N. (1994). Developing an Instrument to Measure Customer Service Quality in Branch Banking. International Journal of Bank Marketing, 12(6), 10-18. https://doi.org/10.1108/02652329410063223

Awan, M., Bukhari, S., \& Iqbal, A. (20 de Septiembre de 2011). Service quality and customer satisfaction in the banking sector: A comparative study of conventional and Islamic banks in Pakistan. Journal of Islamic Marketing, 2(3), 203-224. doi:10.1108/17590831111164750

Bahia, K., \& Nantel, J. (2000). A reliable and valid measurement scale for the perceived service quality of banks. International Journal of Bank Marketing, 18(2), 84-91. https://doi.org/10.1108/02652320010322994

Banco Mundial. (20 de Abril de 2018). La inclusión financiera es un factor clave para reducir la pobreza e impulsar la prosperidad. Obtenido de Banco Mundial: https://www.bancomundial.org/es/topic/financialinclusion/overview

Cortés, M. E., \& Iglesias, M. (2005). Generalidades sobre metodología de la investigación. Ciudad del Carmen, Camp.: Universidad Autónoma del Carmen.

Cronin, J. J., \& Taylor, S. A. (1992). Measuring Service Quality: A Reexamination and Extension. Journal of Marketing, 56(3), 55-68. https://doi.org/10.1177/002224299205600304 East,

R. (1997). Consumer Behaviour: Advances and Applications in Marketing. Prentice Hall.

Escobedo Rivera, J. (2004). La lógica en la investigación y el desarrollo del conocimiento demográfico. Scielo, Vol. 10, (40). ISSN 2448-7147

Famiyeh, S., Asante-Darko, D., \& Kwarteng, A. (2018). Service quality, customer satisfaction, and loyalty in the banking sector. International Journal of Quality \& Reliability Management, 35(8), 1546-1567. doi:10.1108/ijqrm-01-2017-0008

Gupta, A., \& Dev, S. (2012). Client satisfaction in Indian banks: An empirical study. Management Research Review, 35(7), 617-636. https://doi.org/10.1108/01409171211238839

Hernández, R., Fernández, C., \& Baptista, P. (1989). Metodología de Investigación. México: McGraw-Hill : Interamericana. 
Hernández, R., Fernández, C., \& Baptista, P. (2010). Metodología de la Investigación. McGraw Hill.

Ishmuhametov, I. (2018). Customer Satisfaction with Banking Services and Its Estimation. Reliability and Statistics in Transportation and Communication, 585-596. doi:10.1007/978-3-319-74454-4_56

Jabnoun, N. and Khalifa, A. (2005), "A customized measure of service quality in the UAE", Managing Service Quality: An International Journal, Vol. 15 No. 4, pp. 374388. https://doi.org/10.1108/09604520510606844

Kaur, M., Sharma, N., \& Kapoor, S. (2012). Customer Satisfaction in Banking Industry: A Gap Analysis Approach. Asia-Pacific Journal of Management Research and Innovation, 8(4), 399-406. doi:10.1177/2319510x13481894

Paul, J., Mittal, A. and Srivastav, G. (2016), "Impact of service quality on customer satisfaction in private and public sector banks", International Journal of Bank Marketing, Vol. 34 No. 5, pp. 606-622. https://doi.org/10.1108/IJBM-03-2015-0030

Prenshaw, P., Kovar, S., \& Gladden, K. (2006). The impact of involvement on satisfaction for new, nontraditional, credence-based service offerings. Journal of services Marketing, 20(7), 439-452. doi:DOI 10.1108/08876040610704874

Rodríguez, A., \& Pérez, A. O. (2017). Métodos científicos de indagación y de construcción del conocimiento. Revista EAN, (82). https://doi.org/10.21158/01208160.n82.2017.1647

Saleh, A., Quazi, A., Keating, B., \& Gaur, S. (2017). Quality and image of banking services: A comparative study of conventional and Islamic banks. International Journal of Bank Marketing. doi:https://doi.org/10.1108/IJBM-08-2016-0111

Subhashini, S., \& Preetha, S. (17 de Septiembre de 2018). An empirical analysis of service quality factors pertaining to ocean freight forwarding services, Maritime Business Review. Emerald, 276-289. Obtenido de https://www.emerald.com/insight/content/doi/10.1108/MABR-01-2018-0004/full/html

Torres, J., \& Luna, I. (Diciembre de 2017). Evaluación de la percepción de la calidad de los servicios bancarios mediante el modelo SERVPERF. doi:https://doi.org/10.1016/j.cya.2016.01.009

Ushanta, C., Wijeratne, A., \& Samantha, P. (Marzo de 2014). Customers' Perception on Service Quality towards Satisfaction: An Application of SERVPERF in State Sector Banks in Sri 
Lanka. European Journal of Business and Management. Obtenido de Research Gate: https://www.researchgate.net/publication/261797224_Customers'_Perception_on_Service _Quality_towards_Satisfaction_An_Application_of_SERVPERF_in_State_Sector_Banks in_Sri_Lanka_European_Journal_of_Business_and_Management

Yilmaz, V., Ari, E., \& Gürbüz, H. (2018). Investigating the relationship between service quality dimensions, customer satisfaction and loyalty in Turkish banking sector. International Journal of Bank Marketing, 36(3), 423-440. doi:10.1108/ijbm-02-2017-0037

Zeithaml, V. A., Berry, L. L., \& Parasuraman, A. (1988). Communication and Control Processes in the Delivery of Service Quality. Journal of Marketing, 52(2), 35-48. https://doi.org/10.1177/002224298805200203 